\title{
Evaluation of histopathological heterogeneity of colorectal cancer liver metastasis sites after preoperative chemotherapy
}

\author{
NOBUHISA MATSUHASHI $^{1 *}$, HIROYUKI TOMITA $^{2 *}$, HIDENORI TANAKA $^{1}$, YOSHINORI IWATA ${ }^{1}$, \\ SATOSHI MATSUI ${ }^{1}$, HISASHI IMAI ${ }^{1}$, MASAHIRO FUKADA ${ }^{1}$, CHIKA MIZUTANI $^{1}$, TAKAO TAKAHASHI ${ }^{1}$, \\ ITARU YASUFUKU ${ }^{1}$, TOMONARI SUETSUGU ${ }^{1}$, RYUTARO MORI ${ }^{1}$, YOSHIHIRO TANAKA ${ }^{1}$, \\ NAOKI OKUMURA $^{1}$, MANABU FUTAMURA ${ }^{1}$ and KAZUHIRO YOSHIDA ${ }^{1}$ \\ Departments of ${ }^{1}$ Surgical Oncology and ${ }^{2}$ Tumor Pathology, \\ Gifu University Graduate School of Medicine, Gifu, Gifu 501-1194, Japan
}

Received March 20, 2021; Accepted July 14, 2021

DOI: $10.3892 / \mathrm{mco} .2022 .2494$

\begin{abstract}
Patients with liver metastases from colorectal cancer (CRLMs) frequently receive chemotherapy prior to liver resection. Histopathological assessment of the resected specimen can evaluate the response to chemotherapy. The present study analyzed the association between histopathological changes in the primary site and liver metastases. The present study comprised 45 patients with resectable CRLMs at the Surgical Oncology Department of Gifu University School of Medicine (Gifu, Japan) between January 2006 and August 2015. The study included 24 men and 21 women. The primary colonic tumor was located in the right side in 13 (28.9\%) patients and the left side in $32(71.9 \%)$ patients. The present study evaluated patients with metastatic colorectal cancer (31/45) after excluding those in whom histopathological heterogeneity between the primary and liver metastasis changed to grade 3 after chemotherapy. The group that underwent hepatectomy after chemotherapy $(n=25)$ was compared with the group that underwent hepatectomy alone $(n=6)$. In 16 $(53.3 \%)$ out of 25 patients, histopathological heterogeneity of the liver metastasis was lost $(\mathrm{P}=0.04)$. In conclusion, chemotherapy appeared to change histopathological heterogeneity. The present study suggested that the histopathological change of intratumoral heterogeneity is reflected by the response to chemotherapy.
\end{abstract}

Correspondence to: Dr Nobuhisa Matsuhashi, Department of Surgical Oncology, Gifu University School of Medicine, Yanagido 1-1, Gifu, Gifu 501-1194, Japan

E-mail: nobuhisa517@hotmail.com

*Contributed equally

Key words: histopathological, heterogeneity, colorectal cancer, liver, metastasis

\section{Introduction}

Colorectal cancer is the third most common human malignant tumor and is one of the major causes of cancer mortality in the Western world. Metastatic tumors account for 40 to $50 \%$ of malignancies in newly diagnosed patients (1). The prognosis of metastatic colorectal cancer (mCRC) remains poor. Among the treatment options for colorectal liver metastases (CRLM), liver resection is the most conducive to a cure, with 5-year overall survival rates of 29-48\%. Even for initially unresectable CRLM, effective chemotherapy, along with targeted therapy, sometimes enables their resection $(2,3)$. Promising treatments for CRLM include chemotherapy and molecular agents that target epidermal growth factor receptor (EGFR) and vascular endothelial growth factor (VEGF). These reports suggest that the combination of targeted agents and chemotherapy can increase rates of liver resection and response, thus improving progression-free and overall survival of patients with CRLM. However, no studies have compared histopathological type by treatment with anti-VEGF agents and anti-EGFR agents for wild-type $R A S$ liver-limited CRLM (4-6).

The present study aimed to compare histopathological types and heterogeneity of colorectal cancer liver metastasis under different treatment in patients with liver-limited CRLM treated with mFOLFOX6 plus anti-VEGF agents vs. mFOLFOX6 plus anti-EGFR agents

\section{Materials and methods}

Patient. This study included 45 patients with CRLM confirmed to be resectable following neoadjuvant chemotherapy (NACT). Patients were treated with surgery alone or with surgery following FOLFOX alone or FOLFOX plus anti-EGFR (cetuximab or panitumumab) FOLFOX plus anti-VEGF (bevacizumab) as first-line treatment at the Surgical Oncology Department of Gifu University School of Medicine (Gifu City/Japan) from January 2006 to August 2015.

Because an important consequence of intratumoral heterogeneity is potential differences in histopathology between primary tumors and their liver metastases, the 
histopathological profile of the primary colorectal tumors prior to and after chemotherapy and that of the CRLMs resected post-chemotherapy were assessed to investigate the changes between them (Fig. 1A and B).

Treatment. In accordance with the Response Evaluation Criteria in Solid Tumors (RECIST) version 1.1 (6), the same methods were used to perform tumor assessment at baseline and subsequently every 8-12 weeks using torso contrast-enhanced computed tomography and liver contrast-enhanced magnetic resonance imaging. The tumor histopathological response rate was defined as the proportion of patients with grade Ib or more necrosis in accordance with the following definition: Grade 0: No necrosis in the tumor; grade 1a: necrosis in $<33.3 \%$ of the tumor; grade $1 \mathrm{~b}$ : necrosis in 33.3-66.6\% of the tumor; grade 2: necrosis in $66.6-<100 \%$ of the tumor; and grade 3: necrosis in $100 \%$ of the tumor. Patients underwent liver resections if their CRLMs were considered resectable, based on tumor assessments performed after receiving at least six cycles of treatment. Liver resection was performed at least 42 days after the last dose of bevacizumab.

We obtained written informed consent from all patients enrolled in this study. The study protocol conformed to the ethical guidelines of the 1975 Declaration of Helsinki. All study procedures involving humans were conducted in accordance with the ethical standards required by our institution, and the national research committee, and were approved by the Institutional Review Board of the Gifu University Graduate School of Medicine (approval no. 28-508; March 23, 2017).

Pathological assessment of primary tumor and CRLMs. Informed consent for histopathological examination was obtained from all enrolled patients. The postoperative pathological liver resection specimens were fixed in formalin, embedded in paraffin, sectioned into 5-mm-thick slices, and stained with hematoxylin and eosin. In this study, we scored tumor heterogeneity by each histopathology forms.

The slice revisions of primary tumor and matched CRLMs were performed by experienced pathologists (Fig. 2).

Statistical analysis. For continuous variables, the data are summarized as the median with range. For comparisons of variables between groups, Kruskal-Wallis test and $\chi^{2}$ test were used in independent cases. Kruskal-Wallis test was used in for continuous variables. Dunn's post hoc test was performed for comparisons between groups. Additionally, the $\chi^{2}$ test was used for categorical variables. A P-value of $<0.05$ was considered to indicate statistical significance. Statistical analysis was performed using JMP12 software (SAS Institute Inc.).

\section{Results}

Patient characteristics. The study comprised 45 patients with mCRC (24 men, 21 women; mean age, 61.9 \pm 9.4 years). Locations of primary tumor in the 45 patients were the right-sided colon in 13 (28.9\%) patients and the left-sided colon in $32(71.9 \%)$ patients. The group treated with surgery alone included nine patients, whilst those treated with surgery after
FOLFOX alone or plus anti-VEGF or anti-EGFR included 12 patients each. There were no significant differences in the 4 categories (Table I).

Histopathologic heterogeneity at the liver metastasis. The heterogeneity of the histopathology between the primary site and the liver metastasis was significantly different in the group treated with surgery alone compared with the other groups $(\mathrm{P}=0.04)$. However, there were no significant differences between the three groups or each two groups. In addition, tub2 histopathology of appeared to be a predictive marker between primary site and posttreatment liver metastasis specimens from patients with CRLM because the histopathology forms disappeared after each chemotherapy treatment, except for tub2 (Fig. 3A-D). Though this study didn't showed data, we assume that Histopathological change greatly influence long-term survival. We also evaluated patients with CRLM (31/45) after excluding the patients in whom histopathological heterogeneity changed to grade 3 in the liver metastasis after chemotherapy. This study compared the group which underwent hepatectomy after chemotherapy $(n=25)$ with that which underwent hepatectomy alone $(n=6)$. In all six of the latter patients, histopathological heterogeneity at the liver metastasis was maintained after therapy. However, in 16 out of the 25 patients $(53.3 \%)$ who underwent hepatectomy after chemotherapy, histopathological heterogeneity of liver metastases was lost. We confirmed the loss of neoplastic cells by chemotherapy and homogeneity in liver metastases $(\mathrm{P}=0.04)$ (Table II).

\section{Discussion}

The present study describes the histopathological patterns of response of CRLMs to preoperative NACT followed by liver resection. To our knowledge, this is the first report to focus on change of histopathological heterogeneity comparing between primary tumor sites and CRLMs.

Over the past decade, NACT has been widely recommended for the management of initially resectable CRLMs with the aim of inducing tumor shrinkage to identify optimal candidates for subsequent surgical removal. The assessment of tumor regression has been gradually used to quantify the histopathological response to NACT and has served as an early parameter predicting prognosis $(7,8)$.

Discrepancies in the tumor regression patterns in response to different chemotherapy regimens have been revealed in previous literature. Rubbia-Brandt et al $(9,10)$ reported that an oxaliplatin-based regimen improved histopathological response compared with 5-fluorouracil-, and irinotecan-based regimens. In terms of monoclonal antibodies, a bevacizumab-containing regimen provided a better histopathological response than chemotherapy alone or in combination with cetuximab $(11,12)$.

Poultsides et al (13) published a large retrospective analysis of 366 patients (68\% treated preoperatively and $32 \%$ not) who underwent CRLM resection. In that study there was no increase in the degree of necrosis after chemotherapy (13). Nevertheless, it should be noted that only 69 out of 249 (28\%) patients received bevacizumab as part of the preoperative treatment, and the results in 
A

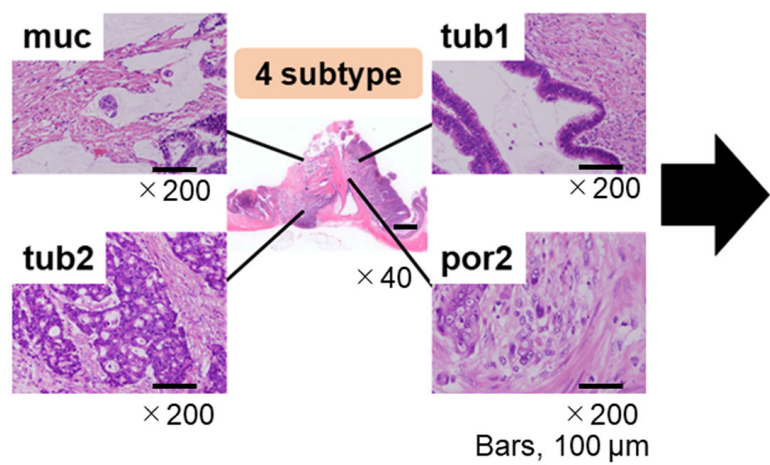

B

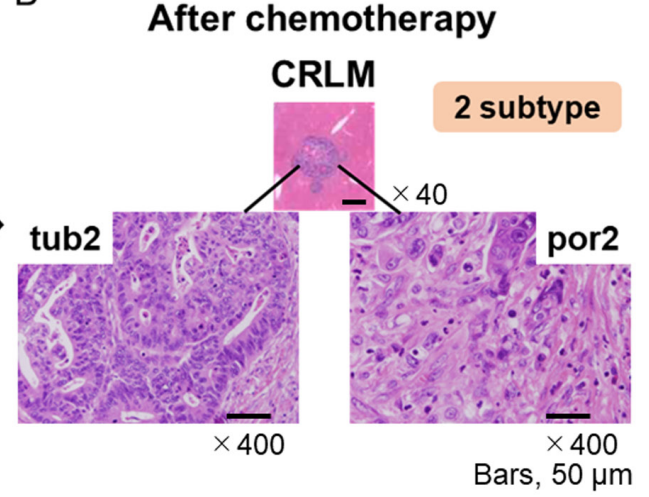

Figure 1. Tumor heterogeneity compared with primary site and metastatic site. (A) Tumor heterogeneity exhibited by a primary colorectal tumor [hematoxylin and eosin stain; magnification, x40 (4 subtype) and x200 (muc, tub1, tub2, por2)]. (B) Tumor heterogeneity of CRLM after chemotherapy. [hematoxylin and eosin stain; magnification, x40 (CRLM) or x400 (tub2, por2)]. CRLM, colorectal liver metastasis; muc, mucinous adenocarcinoma; tub, tubular adenocarcinoma; por, poorly differentiated adenocarcinoma.

terms of necrosis for that subgroup were not reported. In other experience, increase in necrosis seemed to be due to a bevacizumab-related effect. Additionally, the tumor histopathological response rate was defined as the proportion of patients with grade $\geq \operatorname{Ib}(14,15)$.

Taken together, all of the above-reported observations of reduced viable cells, fibrosis, and necrosis from a pathological perspective explained the typical pattern of CLMs detected using computed tomography scanning in patients receiving chemotherapy and bevacizumab. Before treatment, the lesions showed different types of enhancement, a heterogeneous degree of attenuation, and ill-defined borders that were transformed into hypo-attenuated and homogeneous metastases with well-defined borders after treatment (16). Such histological and morphological characteristics strengthen the hypothesis that the RECIST criteria are not completely adequate for evaluating response in patients receiving bevacizumab (17).

In contrast, the anti-EGFR agent cetuximab in combination with chemotherapy has been reported to increase the response rate and yield a good curative hepatectomy. In the PEAK (18), FIRE-3 (19) and CALGB/SWOG 80405 (20) randomized controlled trials performed to compare bevacizumab and anti-EGFR therapy for the progression of recurrent $\mathrm{CRC}$, anti-EGFR was also confirmed to have a positive effect on survival extension in the presence of wild-type RAS.

Recently, the multicenter, randomized, phase II ATOM trial from Japan was designed to evaluate the efficacy and safety of mFOLFOX6 plus bevacizumab and mFOLFOX6 plus cetuximab in patients with liver-limited metastasis from wild-type all-RAS CRC. After study treatment followed by surgical resection of tumors with R0/R1 status, the median progression-free survival of the bevacizumab-treated arm was 6.5 months [95\% confidence interval $(\mathrm{CI})=4.0-13.6$ months], whereas that of the cetuximab-treated arm was 13.8 months $(95 \% \mathrm{CI}=8.4$ months-not reached; hazard ratio $=0.610,95 \% \mathrm{CI}=0.298-1.245)$. Of the 57 tumors for which the histopathological analysis was assessable, the histopathological response rate (grade $1 \mathrm{~b} / 2 / 3$ ) was $66.6 \%(20 / 30)$ in the bevacizumab-treated arm and $92.6 \%$

\section{Scoring of tumor heterogeneity}

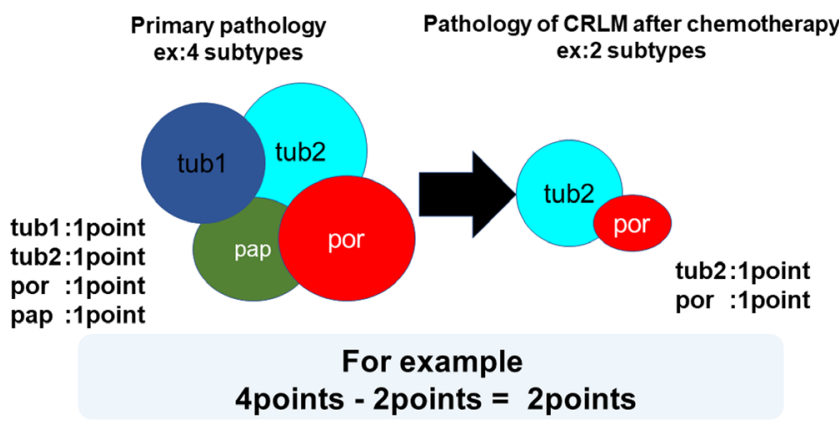

Figure 2. Scoring of tumor heterogeneity. Schema for assessing tumor heterogeneity in primary colorectal tumors and CRLM after chemotherapy. This study counted each of the organisational types as one point. For example, points: Primary site: tub1, tub2, muc and por (total 4points)-metastatic site: por 2 and tub2 (total 2 points) $=2$ points. CRLM, colorectal liver metastasis; ex example; tub1, well differentiated tubular adenocarcinoma; tub2, moderately differentiated tubular adenocarcinoma; por, poorly differentiated adenocarcinoma; pap, papillary adenocarcinoma.

$(25 / 27)$ in the cetuximab-treated arm $(\mathrm{P}=0.0229)(16)$, indicating that the rate tended to be better in the cetuximab-treated arm (21).

Falcão et al (22) reported three categories of tumor growth: i) Replacement growth pattern, in which the tumor permeates between the liver hepatocytes without disrupting the normal architecture; ii) desmoplastic growth pattern, in which the tumor is separated from the liver parenchyma by a band of fibrous tissue that contains tumor-infiltrating lymphocytes; and iii) pushing growth pattern, in which the tumor expands and compresses the surrounding hepatocytes. They reported the pushing growth pattern to be an independent risk factor for reduced survival (22).

Recently, a tumor-heterogeneity concept that considers a single tumor to consist of many tumor cell sub-clones has become an important topic in cancer genomics (23). It is hypothesized to play a critical role in the progression of many cancer types and is a major obstacle to precision cancer therapy. During this process, sub-clones continuously arise via 
Table I. Patient characteristics.

\begin{tabular}{|c|c|c|c|c|c|}
\hline Characteristic & $\begin{array}{l}\text { Surgery only } \\
\qquad(\mathrm{n}=9)\end{array}$ & $\begin{array}{l}\text { FOLFOX } \\
\quad(n=12)\end{array}$ & $\begin{array}{c}\text { Anti-EGFR + FOLFOX } \\
(n=12)\end{array}$ & $\begin{array}{l}\text { Anti-VEGF + FOLFOX } \\
\qquad(n=12)\end{array}$ & P-value \\
\hline \multicolumn{6}{|l|}{ Sex, $n$} \\
\hline Male & 3 & 10 & 5 & 6 & \multirow[t]{2}{*}{0.08} \\
\hline Female & 6 & 2 & 7 & 6 & \\
\hline \multicolumn{6}{|l|}{ Age, years } \\
\hline Median (range) & $65.7(50-81)$ & $64.8(49-83)$ & $59.5(49-68)$ & $58.7(40-68)$ & 0.45 \\
\hline \multicolumn{6}{|l|}{ Location, $\mathrm{n}$} \\
\hline Right side & 2 & 3 & 3 & 5 & \multirow[t]{2}{*}{0.78} \\
\hline Left side & 7 & 9 & 9 & 7 & \\
\hline \multicolumn{6}{|l|}{ RECIST, n } \\
\hline $\mathrm{SD}$ & - & 3 & 2 & 4 & \multirow[t]{3}{*}{0.27} \\
\hline PR & - & 9 & 10 & 8 & \\
\hline $\mathrm{CR}$ & - & 0 & 0 & 0 & \\
\hline \multicolumn{6}{|l|}{ Grade $^{\mathrm{a}}, \mathrm{n}$} \\
\hline $1 \mathrm{a} / 1 \mathrm{~b}$ & & 3 & 2 & 2 & \multirow[t]{3}{*}{0.37} \\
\hline 2 & & 9 & 8 & 8 & \\
\hline 3 & & 0 & 2 & 2 & \\
\hline \multicolumn{6}{|l|}{ Histopathology, $\mathrm{n}$} \\
\hline \multicolumn{6}{|l|}{ Primary } \\
\hline Heterogenous & 6 & 7 & 11 & 12 & \multirow[t]{2}{*}{0.04} \\
\hline Homogenous & 3 & 5 & 1 & 0 & \\
\hline \multicolumn{6}{|l|}{ Liver } \\
\hline Heterogenous & 6 & 4 & 6 & 5 & \multirow[t]{2}{*}{0.17} \\
\hline Homogenous & 3 & 8 & 6 & 7 & \\
\hline
\end{tabular}

${ }^{a}$ Of metastases only. Heterogeneity of differences in pathology between the colorectal primary tumors and the colorectal liver metastases after surgery only, and chemotherapy with FOLFOX alone and with antibody to VEGF or EGFR. VEGF, vascular endothelial growth factor; EGFR, epidermal growth factor receptor; CR, complete response; PR, partial response; RECIST, Response Evaluation Criteria in Solid Tumors (5); $\mathrm{SD}$, stable disease.

Table II. Evaluation of histopathologic heterogeneity in patients who received hepatectomy after chemotherapy versus without chemotherapy.

\begin{tabular}{lccc}
\hline Treatment & Homogeneous & Heterogeneous & P-value \\
\hline $\begin{array}{l}\text { Hx after Cx, } \\
\mathrm{n}(\%)(\mathrm{n}=25)\end{array}$ & $11 / 25(44)$ & $14 / 25(56)$ & 0.03 \\
$\begin{array}{l}\text { Hx alone, } \\
\mathrm{n}(\%)(\mathrm{n}=6)\end{array}$ & $0 / 6(0)$ & $6 / 6(100)$ & \\
\hline
\end{tabular}

$\mathrm{Hx}$, hepatectomy; $\mathrm{Cx}$, chemotherapy.

genomic mutation. The presence of sub-clones has been shown to adversely affect outcome in chronic lymphocytic leukemia, head and neck cancer, and lung adenocarcinoma. However, the full complement of factors that lead to tumor heterogeneity during CRC progression is unknown.

Several promising CRLM treatments have been reported, including chemotherapy and molecular agents that target
EGFR and VEGF. Anti-EGFR drugs resulted in high response and resection rates in the CELIM phase II trial and other studies for initially unresectable CRLM with wild-type KRAS (24,25). Anti-VEGF regimens, such as mFOLFOX6 or CAPEOX plus bevacizumab, have also shown high response and resection rates in phase II studies (19). These reports suggest that the combination of targeted agents and chemotherapy can increase the response rate.

A previous study reported a higher pathological response rate to bevacizumab than for cetuximab. This study suggested that the loss of intratumoral heterogeneity markedly affects the response to chemotherapy. But this study suggested that the heterogeneity of the histopathology between the primary site and the liver metastasis was significantly different in the group treated with surgery alone compared with the other groups $(\mathrm{P}=0.04)$. However, there were no significant differences between the three groups.

In conclusion, the present study highlighted marked differences between pre and posttreatment specimens from sites in patients with mCRC. Tub2 of histopathological type appeared to be a predictive marker in specimens comparing primary site and CRLMs posttreatment because histopathology types 
A

C
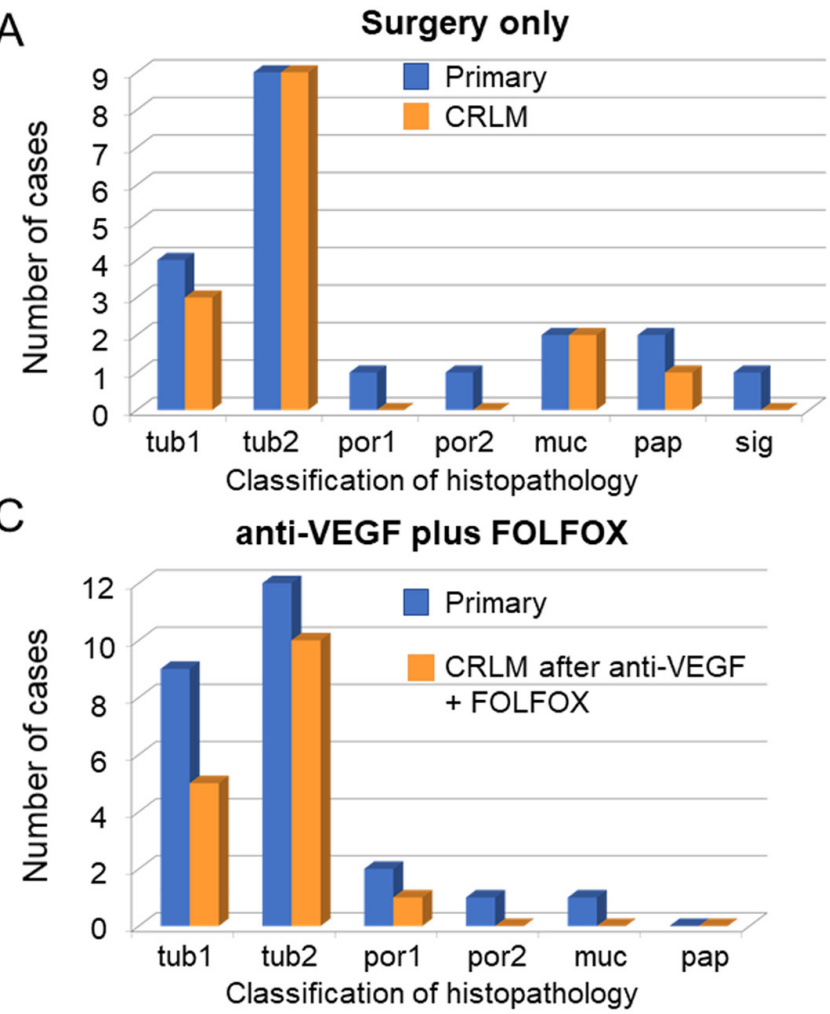

B

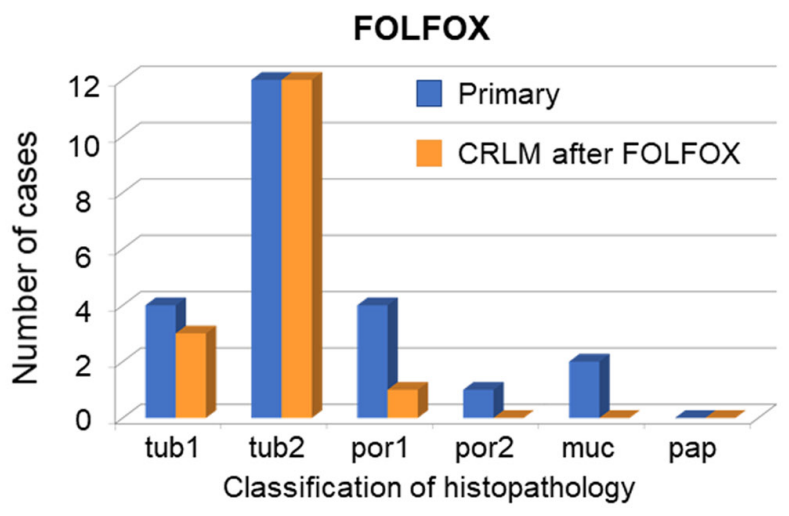

$\mathrm{D}$

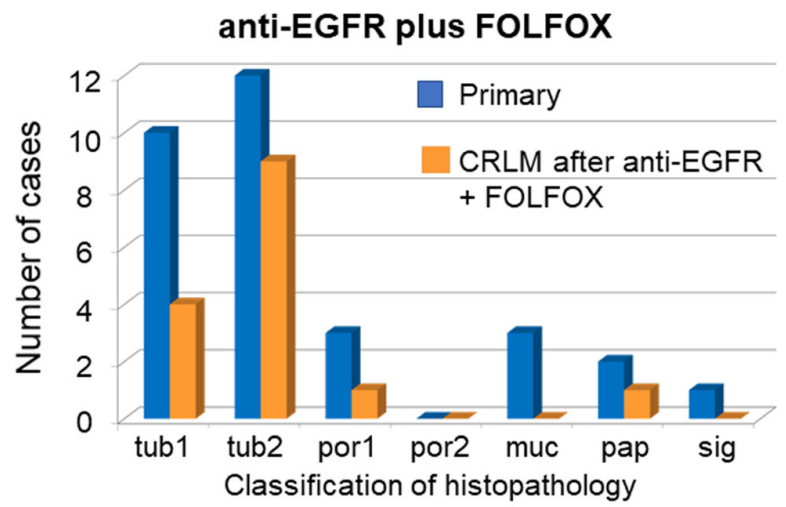

Figure 3. Comparison of the classification of histopathology primary colorectal tumors and CRLM. (A) Comparison of the classification of histopathology primary colorectal tumors and CRLM in patients treated with surgery only. (B) After treatment with FOLFOX. (C) After treatment with antibody to anti-VEGF plus FOLFOX. (D) After treatment with antibody to anti-EGFR plus FOLFOX. CRLM, colorectal liver metastasis; VEGF, vascular endothelial growth factor; EGFR, epidermal growth factor receptor; tub, tubular; por, poorly; muc, mucinous; pap, papillary; sig, signet.

other than tub2 disappeared after chemotherapy treatment. Each NACT agent had an acceptable safety profile. In the near future, we expect results from further study to expand the indication for NACT.

\section{Acknowledgements}

Not applicable.

\section{Funding}

No funding was received.

\section{Availability of data and materials}

The datasets used and/or analyzed during the current study are available from the corresponding author on reasonable request.

\section{Authors' contributions}

NM, HTo and TT conceived the study and its design. NM, HTa, TT, YI, MFuk, IY, TS, CM, RM, HI, YT, NO, MFut and KY acquired the data. NM, HTo and SM analyzed and interpreted the data and drafted the article. NM, HTo, MFut and KY performed critical revision of the article. HTo, MFut and KY supervised the study. NM and HTo confirm the authenticity of all the raw data. All authors read and approved the final manuscript.

\section{Ethics approval and consent to participate}

Written informed consent was obtained from all patients enrolled in the present study. The study protocol conformed to the ethical guidelines of the 1975 Declaration of Helsinki and the guidelines of the regional ethical committees of Zurich and Basel, Switzerland, and was approved by the Institutional Review Board of the Gifu University Graduate School of Medicine (approval no. 28-508; March 23, 2017; Gifu, Japan).

\section{Patient consent for publication}

Not applicable.

\section{Competing interests}

KY has received honoraria for lectures from Chugai Pharmaceutical Co., Ltd., Taiho Pharmaceutical Co., Ltd., Takeda Pharmaceutical Co., Ltd., Eli Lilly and Company, Daiichi Sankyo Co., Ltd., Ono Pharmaceutical Co., Ltd., Merck Serono Co., Ltd., Novartis Pharma K.K., and Sanofi K.K.; and research funding from Ajinomoto Pharmaceutical Co., Ltd., Takeda Pharmaceutical Co., Ltd., Chugai Pharmaceutical Co., Ltd., Daiichi Sankyo Co., Ltd., Taiho Pharmaceutical Co., Ono Pharmaceutical Co., and Yakult Honsha Co., Ltd. outside the submitted work. TT has received honoraria for lectures from Takeda Pharmaceutical Co., Ltd. All remaining authors declare that they have no competing interests. 


\section{References}

1. Sung H, Ferlay J, Siegel RL, Laversanne M, Soerjomataram I, Jemal A and Bray F: Global cancer statistics 2020: GLOBOCAN estimates of incidence and mortality worldwide for 36 cancers in 185 countries. CA Cancer J Clin 71: 209-249, 2021.

2. Cancer Statistics in Japan-2018. Foundation for Promotion of Cancer Research. Available from: https://ganjoho.jp/reg stat/statistics/brochure/backnumber/2018_jp.html.

3. NCCN GUIDELINES FOR PATIENTS 2018. Available from: https://www.nccn.org/patients/guidelines/content/PDF/ colon-patient.pdf.

4. Saltz LB, Clarke S, Díaz-Rubio E, Scheithauer W, Figer A, Wong R, Koski S, Lichinitser M, Yang TS, Rivera F, et al: Bevacizumab in combination with oxaliplatin-based chemotherapy as first-line therapy in metastatic colorectal cancer: A randomized phase III study. J Clin Oncol 26: 2013-2019, 2008.

5. Eisenhauer EA, Therasse P, Bogaerts J, Schwartz LH, Sargent D, Ford R, Dancey J, Arbuck S, Gwyther S, Mooney M, et al: New response evaluation criteria in solid tumors: Revised RECIST guideline (version 1.1). Eur J Cancer 45: 228-247, 2009.

6. Van Cutsem E, Köhne CH, Hitre E, Zaluski J, Chang Chien CR, Makhson A, D'Haens G, Pintér T, Lim R, Bodoky G, et al: Cetuximab and chemotherapy as initial treatment for metastatic colorectal cancer. N Engl J Med 360: 1408-1417, 2009.

7. Chua TC, Saxena A, Liauw W, Kokandi A and Morris DL: Systematic review of randomized and nonrandomized trials of the clinical response and outcomes of neoadjuvant systemic chemotherapy for resectable colorectal liver metastases. Ann Surg Oncol 17: 492-501, 2010.

8. Nordlinger B, Van Cutsem E, Gruenberger T, Glimelius B, Poston G, Rougier P, Sobrero A and Ychou M; European Colorectal Metastases Treatment Group; Sixth International Colorectal Liver Metastases Workshop: Combination of surgery and chemotherapy and the role of targeted agents in the treatment of patients with colorectal liver metastases: Recommendations from an expert panel. Ann Oncol 20: 985-992, 2009.

9. Rubbia-Brandt L: Hepatic lesions induced by systemic chemotherapy for digestive cancer. Ann Pathol 30: 421-425, 2010 (In French).

10. Rubbia-Brandt L, Giostra E, Brezault C, Roth AD, Andres A, Audard V, Sartoretti P, Dousset B, Majno PE, Soubrane O, et al: Importance of histological tumor response assessment in predicting the outcome in patients with colorectal liver metastases treated with neo-adjuvant chemotherapy followed by liver surgery. Ann Oncol 18: 299-304, 2007.

11. Gruenberger B, Tamandl D, Schueller J, Scheithauer W, Zielinski C, Herbst F and Gruenberger T: Bevacizumab, capecitabine, and oxaliplatin as neoadjuvant therapy for patients with potentially curable metastatic colorectal cancer. J Clin Oncol 26: 1830-1835, 2008

12. Gruenberger B, Schueller J, Heubrandtner U, Wrba F, Tamandl D, Kaczirek K, Roka R, Freimann-Pircher S and Gruenberger T: Cetuximab, gemcitabine, and oxaliplatin in patients with unresectable advanced or metastatic biliary tract cancer: A phase 2 study. Lancet Oncol 11: 1142-1148, 2010.

13. Poultsides GA, Bao F, Servais EL, Hernandez-Boussard T, Dematteo RP, Allen PJ, Fong Y, Kemeny NE, Saltz LB, Klimstra DS, et al: Pathologic response to preoperative chemotherapy in colorectal liver metastases: Fibrosis, not necrosis, predicts outcome. Ann Surg Oncol 19: 2797-2804, 2012.

14. Klinger M, Tamandl D, Eipeldauer S, Hacker S, Herberger B, Kaczirek K, Dorfmeister M, Gruenberger B and Gruenberger T: Bevacizumab improves pathological response of colorectal cancer liver metastases treated with XELOX/FOLFOX. Ann Surg Oncol 17: 2059-2065, 2010
15. Wicherts DA, de Haas RJ, Sebagh M, Saenz Corrales E, Gorden DL, Levi F, Paule B, Azoulay D, Castaing D and Adam R: Impact of bevacizumab on functional recovery and histology of the liver after resection of colorectal metastases. Br J Surg 98: 399-407, 2011.

16. Chun YS, Vauthey JN, Boonsirikamchai P, Maru DM, Kopetz S, Palavecino M, Curley SA, Abdalla EK, Kaur H, Charnsangavej C and Loyer EM: Association of computed tomography morphologic criteria with pathologic response and survival in patients treated with bevacizumab for colorectal liver metastases. JAMA 302: 2338-2344, 2009.

17. Gruenberger T, Arnold D and Rubbia-Brandt L: Pathologic response to bevacizumab-containing chemotherapy in patients with colorectal liver metastases and its correlation with survival. Surg Oncol 21: 309-315, 2012.

18. Schwartzberg LS, Rivera F, Karthaus M, Fasola G, Canon JL, Hecht JR, Yu H, Oliner KS and Go WY: PEAK: A randomized, multicenter phase II study of panitumumab plus modified fluorouracil, leucovorin, and oxaliplatin (mFOLFOX6) or bevacizumab plus mFOLFOX6 in patients with previously untreated, unresectable, wild-type KRAS exon 2 metastatic colorectal cancer. J Clin Oncol 32: 2240-2247, 2014.

19. Heinemann V, von Weikersthal LF, Decker T, Kiani A, Vehling-Kaiser U, Al-Batran SE, Heintges T, Lerchenmüller C, Kahl C, Seipelt G, et al: FOLFIRI plus cetuximab versus FOLFIRI plus bevacizumab as first-line treatment for patients with metastatic colorectal cancer (FIRE-3): A randomised, open-label, phase 3 trial. Lancet Oncol 15: 1065-1075, 2014.

20. Venook AP, Niedzwiecki D, Lenz HJ, Innocenti F, Fruth B, Meyerhardt JA, Schrag D, Greene C, O'Neil BH, Atkins JN, et al: Effect of first-line chemotherapy combined with cetuximab or bevacizumab on overall survival in patients with KRAS wild-type advanced or metastatic colorectal cancer: A randomized clinical trial. JAMA 317: 2392-2401, 2017.

21. Oki E, Emi Y, Yamanaka T, Uetake H, Muro K, Takahashi T, Nagasaka T, Hatano E, Ojima H, Manaka D, et al: Randomised phase II trial of mFOLFOX6 plus bevacizumab versus mFOLFOX6 plus cetuximab as first-line treatment for colorectal liver metastasis (ATOM trial). Br J Cancer 121: 222-229, 2019.

22. Falcão D, Alexandrino H, Caetano Oliveira R, Martins J, Ferreira L, Martins R, Serôdio M, Martins M, Tralhão JG, Cipriano MA, et al: Histopathologic patterns as markers of prognosis in patients undergoing hepatectomy for colorectal cancer liver metastases-Pushing growth as an independent risk factor for decreased survival. Eur J Surg Oncol 44: 1212-1219, 2018.

23. Li H, Courtois ET, Sengupta D, Tan Y, Chen KH, Goh JJL, Kong SL, Chua C, Hon LK, Tan WS, et al: Reference component analysis of single-cell transcriptomes elucidates cellular heterogeneity in human colorectal tumors. Nat Genet 49: 708-718, 2017.

24. Folprecht G, Gruenberger T, Bechstein WO, Raab HR, Lordick F, Hartmann JT, Lang H, Frilling A, Stoehlmacher J, Weitz J, et al: Tumor response and secondary resectability of colorectal liver metastases following neoadjuvant chemotherapy with cetuximab: The CELIM randomised phase 2 trial. Lancet Oncol 11: 38-47, 2010.

25. Rivera F, Karthaus M, Hecht JR, Sevilla I, Forget F, Fasola G, Canon JL, Guan X, Demonty G and Schwartzberg LS: Final analysis of the randomised PEAK trial: Overall survival and tumor responses during first-line treatment with mFOLFOX6 plus either panitumumab or bevacizumab in patients with metastatic colorectal carcinoma. Int J Colorectal Dis 32: 1179-1190, 2017.

This work is licensed under a Creative Commons Attribution-NonCommercial-NoDerivatives 4.0 International (CC BY-NC-ND 4.0) License. 